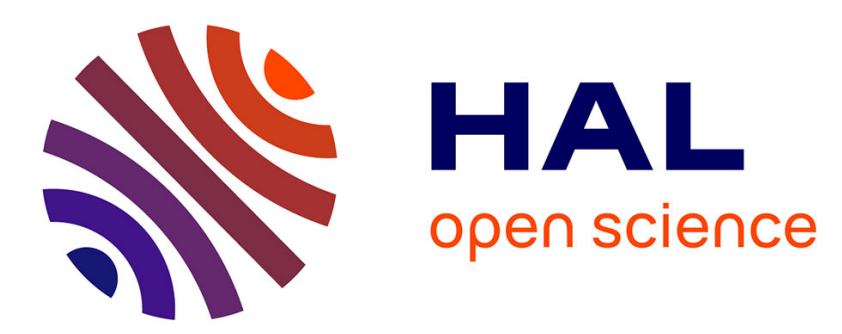

\title{
Caractérisation par magnétotransport d'une couche électronique bidimensionnelle dans une structure GaAs à dopage Si dans un plan (100)
}

A. Zrenner, F. Koch, J. Galibert, Michel Goiran, Jean Léotin, K. Ploog, G. Weimann

\section{To cite this version:}

A. Zrenner, F. Koch, J. Galibert, Michel Goiran, Jean Léotin, et al.. Caractérisation par magnétotransport d'une couche électronique bidimensionnelle dans une structure GaAs à dopage Si dans un plan (100). Revue de Physique Appliquée, 1989, 24 (1), pp.31-35. 10.1051/rphysap:0198900240103100 . jpa-00246024

\section{HAL Id: jpa-00246024 https://hal.science/jpa-00246024}

Submitted on 1 Jan 1989

HAL is a multi-disciplinary open access archive for the deposit and dissemination of scientific research documents, whether they are published or not. The documents may come from teaching and research institutions in France or abroad, or from public or private research centers.
L'archive ouverte pluridisciplinaire HAL, est destinée au dépôt et à la diffusion de documents scientifiques de niveau recherche, publiés ou non, émanant des établissements d'enseignement et de recherche français ou étrangers, des laboratoires publics ou privés. 


\title{
Caractérisation par magnétotransport d'une couche électronique bidimensionnelle dans une structure GaAs à dopage Si dans un plan (100)
}

\author{
A. Zrenner $\left({ }^{1}\right)$, F. Koch $\left({ }^{1}\right)$, J. Galibert $\left({ }^{2}\right)$, M. Goiran $\left({ }^{2}\right)$, J. Leotin $\left({ }^{2}\right)$, \\ K. Ploog $\left({ }^{3}\right)$ and G. Weimann $\left({ }^{4}\right)$ \\ (1) Physik Department T.U.M. D. 8046 Garching, F.R.G. \\ ( ${ }^{2}$ ) Laboratoire Physique des Solides et Service des Champs magnétiques Intenses, I.N.S.A., \\ F. 31077 Toulouse Cedex, France \\ (3) Max-Planck Institut für Festkörperforschung D. 7000 Stuttgart 80, F.R.G. \\ $\left({ }^{4}\right)$ Forschungsinstitut der Deutschen Bundespost D. 6100 Darmstadt, F.R.G.
}

(Reçu le $1^{\text {er }}$ juillet 1988, accepté le 20 septembre 1988)

\begin{abstract}
Résumé. - Un gaz bidimensionnel d'électrons, obtenu par dopage au silicium dans le plan (100) durant la croissance épitaxiale par jet moléculaire de GaAs, est caractérisé à $2 \mathrm{~K}$ par l'étude de l'effet Hall et de la magnétorésistance jusqu'à $43 \mathrm{~T}$. Lorsque le champ magnétique est perpendiculaire au plan (100), trois sousbandes électriques, avec des concentrations respectives de 3,$6 ; 1,18$ et $0,48 \times 10^{12} \mathrm{~cm}^{-2}$ sont révélées expérimentalement. Elles rendent compte de $90 \%$ de la population des atomes de silicium et présentent des mobilités de $780 ; 5200$ et $8200 \mathrm{~cm}^{2} /$ V.s. Une épaisseur de la couche égale à $30 \AA ̊$ a pu être calculée. Lorsque le champ magnétique est parallèle au plan de la couche, la magnétorésistance donne une signature des sousbandes électriques qui révèle une quatrième sous-bande peuplée. Enfin, l'effet Hall quantique est mis en évidence pour la première fois dans un système électronique de mobilité inférieure à $1000 \mathrm{~cm}^{2} / \mathrm{V} . \mathrm{s}$.
\end{abstract}

\begin{abstract}
GaAs}$ prepared by molecular beam epitaxy are characterized by magnetotransport measurements at $2 \mathrm{~K}$ up to $43 \mathrm{~T}$. Shubnikov-de Haas measurements on the magnetoresistance give evidence of three electric subbands, having respectively the populations $3.6 ; 1.18$ and $0.48 \times 10^{12} \mathrm{~cm}^{-2}$. The measured electronic concentrations account for $90 \%$ of the total Silicon donors introduced during the growth. In addition, the doping layer thickness is estimated equal to $30 \AA$. Low field magnetotransport measurement enable to derive the subbands mobility values found respectively equal to $780 ; 5200$ and $8200 \mathrm{~cm}^{2} / \mathrm{V}$.s. Finally, the quantum Hall effect is shown for the first time in a 2 DEG having a mobility value below $1000 \mathrm{~cm}^{2} / \mathrm{V} . \mathrm{s}$.
\end{abstract}

\section{Introduction.}

Le dopage dans un plan cristallin du GaAs est réalisé en épitaxie par jet moléculaire selon le procédé de croissance interrompue [1]. Pour obtenir un dopage de type $\mathrm{n}$, des atomes de Si sont introduits sur une surface terminée par un plan d'atomes d'arsenic tandis que le flux de gallium est interrompu. La reprise de la croissance du cristal conduit en principe à enterrer les dopants $\mathrm{Si}$ sur des sites gallium situés dans un même plan atomique. En réalité, le dopage dans un plan unique n'est jamais réalisé et les atomes de Si sont répartis dans une couche d'épaisseur finie $\mathrm{d} z$ qui dépend des paramètres de la croissance [2]. On obtient toutefois selon ce procédé un gaz bidimensionnel (2D) d'électrons lorsque la densité d'atomes dopants est suffisante pour donner un gaz dégénéré. La distance moyenne entre les impuretés est alors inférieure au rayon de Bohr. Cela donne une concentration minimale de $2 \times 10^{11} \mathrm{~cm}^{-2}$ pour une couche atomique de dopants. Le potentiel attractif des impuretés dans le plan, entourées du gaz électronique, engendre un spectre de sous-bandes électriques caractéristique d'un gaz 2D. L'écart énergétique entre les sous-bandes est d'ailleurs déterminé par l'épaisseur de la couche dopée pour une concentration surfacique donnée [3].

Le magnétotransport à fort champ magnétique permet la détermination de la concentration électro- 
nique, des populations et des mobilités des diverses sous-bandes électriques occupées, et donne aussi l'épaisseur de la couche dopée. Il faut remarquer qu'une épaisseur de quelques monocouches dopées ne peut être aisément mesurée par les techniques de microscopie. Par ailleurs, la concentration électronique n'est pas accessible par la mesure classique de l'effet Hall dans ces systèmes où plusieurs bandes de mobilités faibles et très différentes sont occupées.

Dans la suite, nous présentons la structure électronique et la caractérisation par magnétotransport d'un échantillon dopé intentionnellement à $6 \times 10^{12} \mathrm{~cm}^{-2}$. Enfin, nous mesurons l'effet Hall quantique qui se manifeste par des plateaux jusqu'à $43 \mathrm{~T}$.

1. Structure électronique et méthodes de caractérisation.

Un calcul auto-cohérent à une dimension, appliqué à une couche d'épaisseur finie $\mathrm{d} z$ et de population $N_{\mathrm{D}}$, détermine le potentiel, les fonctions d'onde et la structure énergétique [3].

La figure 1 donne les résultats de ce calcul pour $N_{\mathrm{D}}=5,6 \times 10^{12} \mathrm{~cm}^{-2}$, valeur mesurée dans la caractérisation par magnétotransport de l'échantillon. L'épaisseur $\mathrm{d} z$ a été fixée arbitrairement à $5 \AA$.

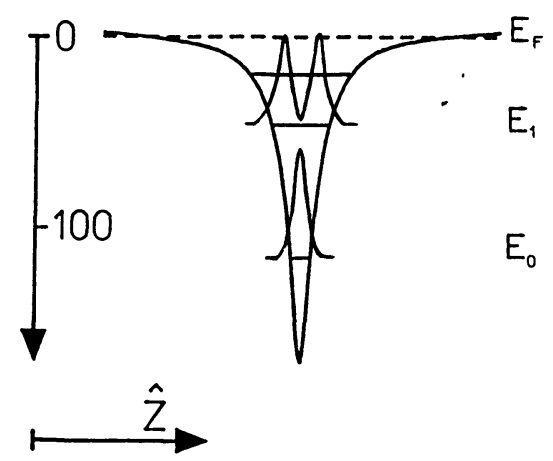

Fig. 1. - Potentiel de Hartree dans la direction perpendiculaire à la croissance dans le cas où $N_{\mathrm{D}}=5,6 \times 10^{12} \mathrm{~cm}^{-2}$ et où l'épaisseur $\mathrm{d} z$ est prise égale à $5 \AA$. Les probabilités de présence pour les sous-bandes $i=0$ et $i=1$ sont représentées.

[Hartree potential in direction perpendicular to the growth direction for a sample with $N_{\mathrm{D}}=5.6 \times 10^{12} \mathrm{~cm}^{-2}$ and $\mathrm{d} z=5 \AA$. Charge distribution in the $i=0$ and $i=1$ subbands.]

Le tableau I donne les énergies $E_{\mathrm{F}}-E_{i}$ ainsi que les densités calculées dans chaque sous-bande. Les mesures ont été réalisées à $2 \mathrm{~K}$ sur un échantillon ayant une géométrie donnant accès simultanément à la magnétorésistance $\mathbf{R x x}$ et à l'effet Hall Rxy. Nous avons utilisé un champ magnétique quasi statique de longue durée (1 s) jusqu'à $43 \mathrm{~T}$ [4].
Tableau I. - Valeurs calculées des énergies $E_{\mathrm{F}}-E_{i}$ et des concentrations pour les quatre premières sousbandes.

[Values of $E_{\mathrm{F}}-E_{i}$ and carrier concentrations calculated for the first four subbands.]

\begin{tabular}{ccc}
\hline$N_{\mathrm{D}}=5,6 \times 10^{12} \mathrm{~cm}^{-2}$ & $E_{\mathrm{F}}-E_{i}(\mathrm{moV})$ & $\begin{array}{c}n_{i} \\
\left(\times 10^{12} \mathrm{~cm}^{-2}\right)\end{array}$ \\
\hline$i=0$ & 115,22 & 3,5 \\
\hline$i=1$ & 43,89 & 1,34 \\
\hline$i=2$ & 17,71 & 0,53 \\
\hline$i=3$ & 0,19 & 0,15
\end{tabular}

Nous rappelons que les populations des sousbandes électriques occupées se déduisent des périodes $\Delta(1 / B)$ mesurées sur le spectre d'oscillation de Shubnikov-de Haas ( $\operatorname{Rxx}$ fonction de $1 / B$ ) par la relation $n \cdot \Delta(1 / B)=e / \pi \hbar$. La mesure de la magnétorésistance $\operatorname{Rxx}(B)$, combinée à l'effet Hall $\operatorname{Rxy}(B)$ donne accès aux valeurs des mobilités dans chaque sous-bande par les relations $\sigma \times \mathbf{x x}=$ $\mathrm{Rxx} /\left(\mathrm{Rxx}^{2}+\mathrm{Rxy}^{2}\right)=\sum_{i} n_{i} \cdot e \cdot \mu_{i} /\left(1+\mu_{i}^{2} B^{2}\right)$.

\section{Résultats expérimentaux et interprétations.}

Sur la figure 2 sont représentées les variations de $\mathbf{R x x}$ et $\mathbf{R x y}$ à $2 \mathrm{~K}$ en fonction du champ magnétique lorsque celui-ci est perpendiculaire au plan dopé. La

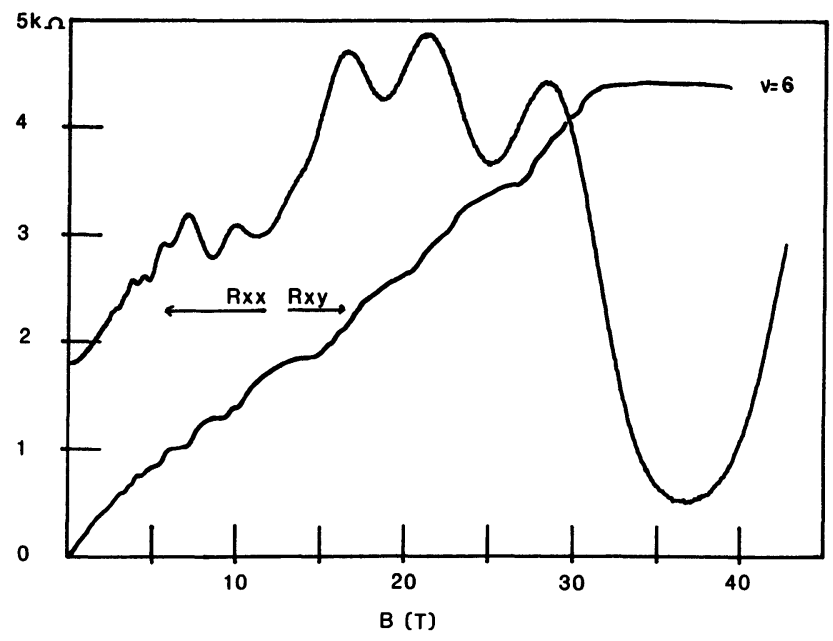

Fig. 2. - Magnétorésistance $\mathrm{Rxx}$ et résistance de Hall $\mathrm{Rxy}$ en fonction du champ magnétique perpendiculaire au plan. $T=2 \mathrm{~K}$.

[Magnetoresistance $\mathbf{R x x}$ and Hall resistance $\mathrm{Rxy}$ as a function of the magnetic field perpendicular to the layer. $T=2 \mathrm{~K}$.] 


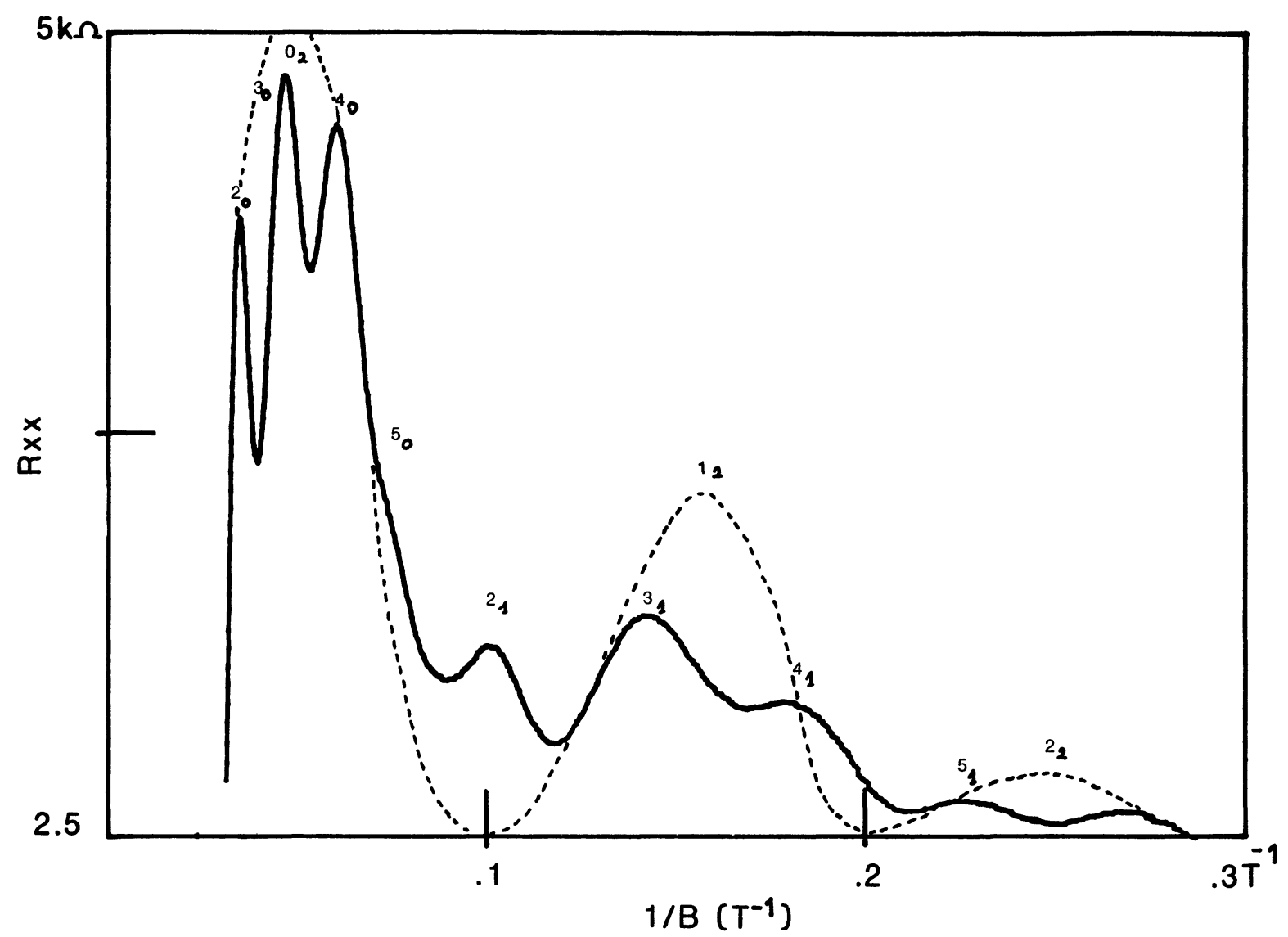

Fig. 3. - Magnétorésistance $\mathrm{Rxx}$ en fonction de l'inverse du champ magnétique perpendiculaire au plan (100). $T=2 \mathrm{~K}$. Les pics de résonance $\mathrm{SdH}$ sont identifiés par $l_{i}$ où $l$ indexe le niveau de Landau et $i$ la sous-bande électrique. En pointillé : tracé des oscillations relatives à la sous-bande $i=2$.

[ $R x x$ as a function of the reciprocal magnetic field perpendicular to the layer. $T=2 \mathrm{~K}$. The $\mathrm{SdH}$ peaks are identified by $l_{i}$ where $l$ labels the $l$-th Landau level and $i$ the $i$-th electric subband. The dotted line is a guideline for the SdH oscillations corresponding to the $i=2$ subband.]

figure 3 montre les variations périodiques de $\operatorname{Rxx}$ en fonction de l'inverse du champ magnétique. L'analyse des résultats de l'effet Shubnikov-de Haas (SdH) met en évidence trois séries d'oscillations. Les concentrations déduites pour chaque sous-bande sont : $n_{0}=3,6 \times 10^{12} \mathrm{~cm}^{-2}, n_{1}=1,28 \times 10^{12} \mathrm{~cm}^{-2}$, $n_{2}=0,52 \times 10^{12} \mathrm{~cm}^{-2}$. La comparaison avec le tableau I permet d'attribuer ces trois séries aux sousbandes $i=0,1$ et 2 . En particulier, la série correspondant à $i=2$ apparaît comme enveloppe du signal. La mise en évidence de la série de la sousbande fondamentale nécessite un champ largement supérieur à $20 \mathrm{~T}$ pour être détectée. La somme $n_{0}+n_{1}+n_{2}$ conduit à une densité totale $N_{\mathrm{s}}=$ $5,4 \times 10^{12} \mathrm{~cm}^{-2}$ qui représente $90 \%$ de la population totale de donneurs introduits durant la croissance. Il faut remarquer que les mesures d'effet Hall à bas champ magnétique $(B<5 \mathrm{~T})$ donnent une concentration de $2,8 \times 10^{12} \mathrm{~cm}^{-2}$, bien inférieure à cette densité. Nous avons analysé la conductivité $\sigma_{\mathbf{x x}}$ comme étant la somme des conductivités de chaque sous-bande [5] et, connaissant les populations de celles-ci, nous en avons déduit les mobilités $\mu_{0}=780 \mathrm{~cm}^{2} / \mathrm{V} . \mathrm{s} ; \mu_{1}=5200 \mathrm{~cm}^{2} / \mathrm{V} . \mathrm{s}$ et $\mu_{2}=$ $8200 \mathrm{~cm}^{2} /$ V.s. Les faibles valeurs de mobilité sont dues à la diffusion par les impuretés ionisées. Cette diffusion est plus intense dans la sous-bande fondamentale où la probabilité de présence dans le plan d'impuretés est maximale. En conséquence, la mobilité dans cette sous-bande est plus faible. Dans les états de sous-bande successivement excités, l'électron est distribué de plus en plus loin du plan d'impuretés, sa mobilité va donc en augmentant.

L'épaisseur $\mathrm{d} z$ est déterminée, comme indiqué précédemment, à partir de la structure énergétique des sous-bandes électriques en fonction de $\mathrm{d} z$ (3), la valeur trouvée est égale à $30 \AA$ avec une barre d'erreur de 10 à $40 \AA$. Pour dz inférieur à $30 \AA$, l'épaisseur de la couche dopée est bien inférieure à l'extension de la fonction d'onde de la sous-bande fondamentale et le spectre énergétique n'est plus sensible à la valeur exacte de l'épaisseur de la couche (3). La caractérisation de l'épaisseur $\mathrm{d} z$ de la couche repose principalement sur la détermination de l'éner- 
gie $E_{\mathrm{F}}-E_{0}$ de la sous-bande fondamentale, dont la valeur est très sensible à l'épaisseur de la couche. De forts champs magnétiques sont alors nécessaires pour révéler les oscillations de cette sous-bande.

Nous avons analysé l'effet Hall dans ce système multibandes jusqu'à $43 \mathrm{~T}$. A bas champ magnétique les porteurs de la sous-bande fondamentale très peu mobiles ne donnent pas de contribution significative à l'effet Hall, bien que cette bande soit la plus peuplée. La pente de l'effet Hall $\operatorname{Rxy}(B)$ traduit le transport des électrons les plus mobiles dans les sous-bandes excitées, qui sont par ailleurs les moins peuplées. A fort champ magnétique, on peut observer un plateau qui s'étend entre 30 et $40 \mathrm{~T}$ à $2 \mathrm{~K}$. La résistance correspondant à ce plateau est égale à $h / 6 e^{2}$. Simultanément la magnétorésistance $\mathrm{Rxx}$ décroît fortement et le minimum de résistance se trouve à $B=37 \mathrm{~T}$. Il est remarquable d'observer l'effet Hall quantique dans un système où les porteurs confinés dans une couche mince dopée, ont une mobilité dans la sous-bande $i=0$ aussi faible que $780 \mathrm{~cm}^{2} / V$.s. C'est en effet le gaz électronique de plus faible mobilité où l'effet Hall quantique ait été mis en évidence jusqu'à ce jour. Le coefficient de remplissage $\nu=6\left(\mathrm{Rxy}=h / 6 e^{2}\right)$ indique que trois niveaux de Landau non résolus en spin sont peuplés. Deux niveaux appartiennent à la sous-bande électrique fondamentale $i=0$ et le troisième à la première sous-bande électrique $i=1$. Les sous-bandes $i=2$ et $i=3$ ne sont cependant que partiellement dépeuplées par le champ magnétique dans la mesure où des états localisés subsistent en dessous de ces sousbandes. Il existe en effet un fond continu de densité d'états indépendante de $B$ évalué à au moins $50 \%$ de la densité d'états à champ nul [6].

Lorsque le champ magnétique est parallèle à la couche dopée, on observe sur la magnétorésistance Rxx une série de points d'inflexions caractéristique de la structure énergétique des sous-bandes. Dans cette configuration, il n'y a pas de quantification de Landau. Le champ magnétique produit par effet diamagnétique un déplacement relatif vers les hautes énergies des sous-bandes électriques d'une quantité $\mathrm{d} E_{i}=e^{2}\left\langle z_{i}^{2}\right\rangle B^{2} / 2 m$. Il en résulte au cours de la croissance du champ magnétique, une redistribution des électrons dans les sous-bandes qui produit un dépeuplement successif des sous-bandes excitées [7]. Lorsqu'une sous-bande traverse le niveau de Fermi, la densité d'état au niveau de Fermi décroît de manière discontinue. Le principal mécanisme de diffusion est la diffusion par impuretés ionisées intra-sous-bandes ou inter-sous-bandes. Au point de transition, quand le bas de la bande traverse le niveau de Fermi, la mobilité augmente du fait de la suppression du mécanisme de diffusion inter-sousbandes ; on prévoit alors une chute abrupte de la résistance. On trouve expérimentalement un point d'inflexion. Les séries d'inflexions correspondent alors au passage de sous-bandes à travers $E_{\mathrm{F}}$. La figure 4 montre la résistance $\mathrm{Rxx}$ lorsque $B$ est parallèle au plan dopé jusqu'à $35 \mathrm{~T}$. On observe deux inflexions situées à 2 et $11 \mathrm{~T}$. Pour les valeurs de champ correspondant à une inflexion, on peut estimer les déplacements diamagnétiques $\mathrm{d} E_{i}$ des sous-bandes et indexer la sous-bande qui traverse le niveau de Fermi. On trouve alors que les inflexions à 2 et $11 \mathrm{~T}$ correspondent aux sous-bandes $i=3$ et $i=2$. La sous-bande $i=3$ estimée à quelques meV en dessous de $E_{\mathrm{F}}$ n'a pas été révélée par les mesures de SdH. Elle est cependant prévue par le calcul de la structure électronique dans lequel on trouve une bande située à $6 \mathrm{meV}$ sous le niveau de Fermi avec une population $n_{3}=0,2 \times 10^{12} \mathrm{~cm}^{-2}$. Le dépeuplement de la sous-bande $i=1$ devrait se produire audelà de $45 \mathrm{~T}$. En résumé, il apparaît que le transport en champ parallèle est un outil simple et puissant pour caractériser le potentiel de Hartree à travers les mesures de $\left\langle z_{i}^{2}\right\rangle$; c'est aussi une méthode permettant de caractériser l'épaisseur de la couche dopée.

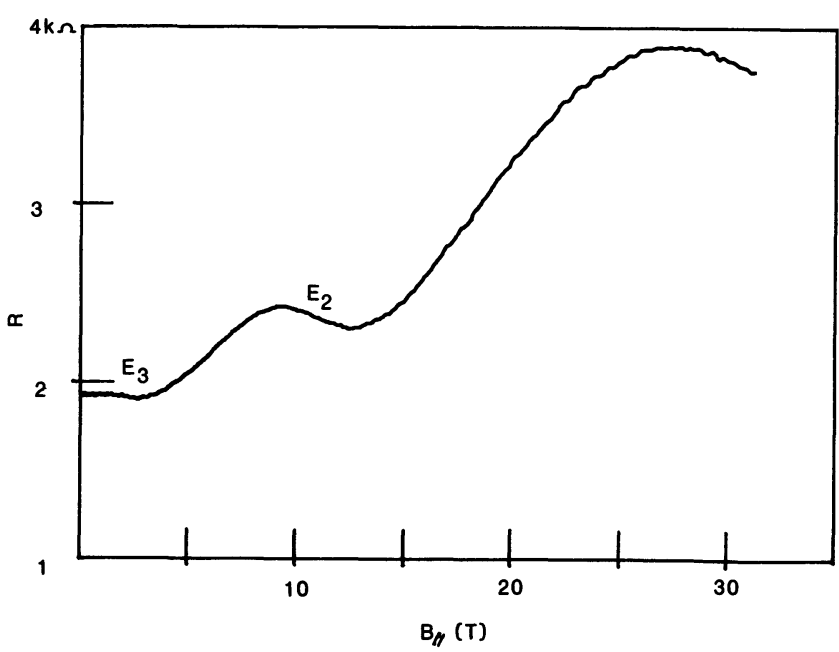

Fig. 4. - Magnétorésistance en fonction du champ magnétique $B$ parallèle au plan (100). $T=2 \mathrm{~K}$. Pour les valeurs 2 et $11 \mathrm{~T}$, il se produit une dépopulation des sous-bandes électriques 3 et 2 respectivement.

[Magnetoresistance $\mathbf{R x x}$ as a function of the magnetic field parallel to the doped layer. $T=2 \mathrm{~K}$. At the field values 2 and $11 \mathrm{~T}$, a depopulation of the $i=3$ and $i=2$ subbands occurs.

\section{Conclusion.}

Le magnétotransport en champ magnétique intense est un moyen unique de caractériser une couche bidimensionnelle obtenue par dopage plan. Les mesures classiques de l'effet Hall et de la résistivité conduisent à des résultats erronés.

La structure électronique des sous-bandes, les concentrations et les mobilités sont obtenues, ainsi que l'épaisseur de la couche. 
La magnétorésistance mesurée avec le champ magnétique dans le plan de la couche a permis de mettre en évidence la série des inflexions associées au changement diamagnétique des énergies des sous-bandes qui traversent le niveau de Fermi et de les indexer.

L'effet Hall quantique est mis en évidence pour la première fois dans un système à deux dimensions de mobilité aussi faible que $780 \mathrm{~cm}^{2} / \mathrm{V}$.s.

Remerciements.

A. Zrenner remercie la société Siemens A.G. pour son support financier.

\section{Bibliographie}

[1] Wood, C. E., Metze, G., Berry, J. and EAstman, L. F., J. Appl. Phys. 51 (1980) 383.

[2] Zrenner, A., KoCH, F. and Ploog, K., Proc. of the 14th Int. Symp. on GaAs and related compounds. Heraklion (1987).

[3] ZrenNER, A. and KoCH, F., Surface Sci. 196 (1988) 671.

[4] Askenazy, S., Fert, Ch., Marquez, J., Bellan, Ph., Wallace, P. R. and Herlach, F., Rev. Phys. Appl. 21 (1986) 563.
[5] Zrenner, A., Reisinger, H., KoCH, F. and Ploog, K., Proc. of the 17th Int. Conf. on the Physics of Semiconductors, San Francisco, 1984, Eds. J. P. Chadi and W. A. Harrison (Springer-Verlag, N. Y. 1985) p. 325.

[6] Weiss, D., Stahl, E., Weimann, G., Ploog, K. and Von KLITZING, K., Surface Sci. 170 (1986) 285.

[7] Zrenner, A., Reisinger, H., Koch, F., PloOG, K. and MAan, J. C., Phys. Rev. B 33 (1986) 5607. 